\title{
EL PROYECTO EMECAM: ESTUDIO MULTICÉNTRICO ESPAÑOL SOBRE LA RELACIÓN ENTRE LA CONTAMINACIÓN ATMOSFÉRICA Y LA MORTALIDAD. ANTECEDENTES, PARTICIPANTES, OBJETIVOS Y MÉTODOLOGÍA *
}

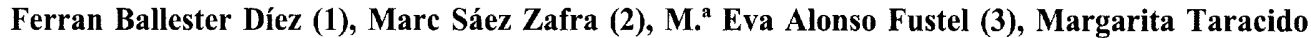 \\ Trunk (4), José M. ${ }^{a}$ Ordóñez Iriarte (5), Inés Aguinaga Ontoso (6), Antonio Daponte Codina (7), \\ Juan Bellido Blasco (8), José Jesús Guillén Pérez (9), M. ${ }^{a}$ José Pérez Boíllos (10), Álvaro Cañada \\ Martínez (11), Federico Arribas Monzón (12) y Santiago Pérez-Hoyos (1) por el Grupo EMECAM. \\ (1) Institut Valencià d'Estudis en Salut Pública (IVESP). Dirección General de Salud Pública. Generalidad Valenciana. \\ (2) Universitat de Girona. Departament d'Economia. \\ (3) Departamento de Sanidad del Gobierno Vasco. \\ (4) Facultad de Medicina de la Universidad de Santiago. \\ (5) Consejería de Sanidad y Servicios Sociales. Comunidad de Madrid. \\ (6) Area de Sanidad y Medioambiente del Ayuntamiento de Pamplona. \\ (7) Escuela Andaluza de Salud Pública. Granada. \\ (8) Centro Salud Pública Area 02 de Castelló. Consellería de Sanidad. \\ (9) Centro Área Cartagena. Consejería de Sanidad de la Comunidad de Murcia. \\ (10) Departamento de Salud y Consumo. Ayuntamiento de Vitoria-Gasteiz. \\ (11) Dirección Regional de Salud Pública. Asturias. \\ (12) Dirección General de Salud Pública. Aragón. \\ (*) Este trabajo cuenta con una beca del Fondo de Investigaciones Sanitarias (Expediente núm 97/0051).
}

\section{RESUMEN}

En los últimos años, un número creciente de estudios sugiere que los incrementos en los niveles de contaminación atmosférica pueden causar efectos a corto plazo sobre la salud, incluso con niveles de contaminación cercanos o inferiores a los considerados hasta ahora como seguros. Los distintos enfoques metodológicos y la diversidad de técnicas de análisis utilizadas han dificultado la comparabilidad directa entre los resultados obtenidos, impidiendo llegar a conclusiones claras. Ello ha estimulado la puesta en marcha de proyectos multicéntricos, como el proyecto APHEA (short-term effects of Air Pollution on Health: a European Approach), dentro del ámbito europeo.

El proyecto EMECAM se enmarca en el contexto de los estudios multicéntricos citados y tiene una proyección amplia en el ámbito nacional español. En él se incluyen 14 ciudades españolas (Barcelona, Gran Bilbao, Cartagena. Castellón, Gijón, Huelva, Madrid, Pamplona, Sevilla, Oviedo, Valencia, Vigo, Vitoria y Zaragoza) que representan diferentes situaciones sociodemográficas, climáticas y ambientales, sumando un total cercano a nueve millones de habitantes.

Correspondencia:

Ferran Ballester Diez.

Institut Valencià d'Estudis en Salut Pública (IVESP).

Juan dc Garay, 21.

46017 Valencia.

Teléfono 96-3869369

Fax 96-3869370

Correo electrónico: fballest@san.gva.es

\section{ABSTRACT \\ The $E M E C A M$ Project: Spanish Multicenter Study on the Relationship Between Air Pollution and the Mortality. Background data, Participants, Objectives and Methods}

\begin{abstract}
In recent years, a growing number of studies suggests that increases in air pollution levels may have short-term impact on human health, even at pollution levels similar to or lower than those which have been considered to be safe to date. The different methodological approaches and the varying analysis techniques employed have made it difficult to make a direct comparison among all of the findings, preventing any clear conclusions from being drawn. This has led to multicenter projects such as the APHEA (Short-Term Impact of Air Pollution on Health. A European Approach) within a European Scope.
\end{abstract}

The EMECAM Project falls within the context of the aforesaid multicenter studies and has a wide-ranging projection nationwide within Spain. Fourteen (14) cities throughout Spain were included in this Project (Barcelona, Metropolitan Area of Bilbao, Cartagena, Castellón, Gijón, Huelva, Madrid, Panılona, Seville, Oviedo, Valencia, Vigo, Vitoria and Saragossa) representing different sociodemographic, climate and environmental situations, adding up to a total of nearly nine million inhabitants. 
El objetivo del proyecto EMECAM es evaluar el impacto a corto plazo de la contaminación atmosférica, en el conjunto de las ciudades participantes, sobre la mortalidad por todas las causas, en toda la población y en personas de 70 y más años, y por causas respiratorias y del aparato circulatorio. Para ello, con un diseño ecológico, se analizan los datos de las series temporales tomando como unidad los datos diarios de mortalidad, contaminantes, temperatura y otros factores, obtenidos de registros de instituciones públicas. El período de estudio, aunque no es exactamente el mismo para todas las ciudades, está comprendido en todos los casos entre los años 1990 y 1996 . El cálculo de las medidas de asociación se realiza mediante regresión autorregresiva de Poisson. En una fase posterior los resultados de cada ciudad se combinarán mediante la realización de un meta-análisis.

Palabras clave: Contaminación atmosférica. Mortalidad. Estudio multicéntrico. Series temporales.
The objective of the EMECAM project is that to asses the short-term impact of air pollution throughout all of the participating cities on the mortality for all causes, on the population and on individuals over age 70, for respiratory and cardiovascular design causes. For this purpose, with an ecological, the time series data analyzed taking the daily deaths, pollutants, temperature data and other factors taken from records kept by public institutions. The period of time throughout which this study was conducted, although not exactly the same for all of the cities involved, runs in all cases from 1990 to 1996. The degree of relationship measured by means of an autoregressive Poisson regression. In the future, the results of each city will be combined by means of a meta-analysis.

Key words: Air pollution. Mortality. Multicenter study. Time series.

\section{INTRODUCCIÓN}

Los efectos sobre la salud de la contaminación atmosférica han sido objeto de un importante número de estudios en la segunda mitad del presente siglo, especialmente a partir de una serie de episodios ocurridos en los países industrializados, entre los que destaca el de Londres en el año 1952, que llevaron al establecimiento de estándares de calidad del aire para la protección de la salud pública ${ }^{1,2}$. En los últimos años, un número creciente de estudios sugiere que los incrementos en los niveles de contaminación atmosférica pueden causar efectos a corto plazo sobre la salud, aún con niveles cercanos o por debajo de los considerados como seguros $^{3-8}$.

Los distintos enfoques metodológicos y la diversidad de técnicas de análisis utilizadas han dificultado la comparabilidad directa entre los resultados obtenidos, impidiendo llegar a conclusiones claras. Ello ha estimulado la puesta en marcha de proyectos multicéntricos como, dentro del ámbito europeo, el proyecto APHEA (short-term effects of Air Pollution on Health: a European Approach $)^{9-11}$. En él se utiliza una extensa base de datos procedente de quince ciudades con diferentes situaciones sociales, climáticas y ambientales, lo que permite una aproximación adecuada al estudio de los efectos a corto plazo de la contaminación atmosférica sobre la salud.
También en España se han llevado a cabo, durante los últimos cinco años, diversos estudios sobre el tema en algunas ciudades $^{7,12-15}$. Sus resultados indican que existe una asociación entre la contaminación atmosférica y la mortalidad y la morbilidad por urgencias respiratorias y los niveles actuales de contaminación atmosférica en las ciudades españolas donde se han realizado. Sin embargo, la metodología aplicada en los estudios citados varía. Además, con la excepción de los realizados en Barcclona, los demás sólo han incluido el dióxido de azufre $\left(\mathrm{SO}_{2}\right)$ y las partículas en suspensión como contaminantes, no habiendo prestado atención a otros, como el ozono $\left(\mathrm{O}_{3}\right)$ y el dióxido de nitrógeno $\left(\mathrm{NO}_{2}\right)$, que pueden ser de importancia en nuestro país. El reto actual se centra, por un lado, en cuantificar adecuadamente los efectos asociados a los niveles moderados y bajos de la contaminación atmosférica y, por otro, en identificar los contaminantes responsables y sus posibles efectos sinérgicos. Todo ello plantea la necesidad de una estandarización de las variables a estudio y una mayor profundización metodológica.

El proyecto $E M E C A M$ se enmarca en el contexto de los estudios multicéntricos citados y tiene una proyección amplia en el ámbito nacional. En él se incluyen 14 ciudades españolas que representan diferentes situaciones sociodemográficas, climáticas y ambientales, sumando entre todas un total cer- 
cano a nueve millones de habitantes (tabla 1). El período de estudio responde a la disponibilidad de datos en cada una de las ciudades, aunque en todos los casos se sitúa entre los años 1990 y 1996 . El período más corto considerado es de 3 años, para garantizar una buena modelización de los componentes de las series, y el más largo es de 5, con el fin de poder asumir estabilidad en las condiciones sociodemográficas de las poblaciones a estudio y en las características de los contaminantes menos específicos (partículas).

El protocolo del proyecto fue sometido a evaluación del Fondo de Investigaciones Sanitarias (FIS) en su convocatoria para 1997. En principio, los grupos participantes correspondían a las doce ciudades siguientes: Barcelona, Gran Bilbao, Cádiz, Cartagena, Castellón, Huelva, Madrid, Pamplona, Sevilla, Valencia, Vigo y Vitoria. Debido a la escasa información disponible sobre con- taminación atmosférica en la ciudad de Cádiz se decidió no incluirla en el presente estudio. Con posterioridad, se adhirieron al proyecto tres nuevas ciudades: Oviedo, Gijón y Zaragoza.

\section{OBJETIVOS}

El objetivo general del proyecto $E M E$ $C A M$ es evaluar el impacto a corto plazo de la contaminación atmosférica sobre la mortalidad diaria por todas las causas, excluyendo las externas, en toda la población y en personas de 70 y más años, por causas respiratorias y por causas del aparato circulatorio, en cada una y en el conjunto de las ciudades participantes. Los objetivos específicos se plantean a dos niveles: en cada grupo y en el conjunto de los grupos. Cada grupo realiza no sólo la recogida de datos sino todas las fases de la investigación en su propia ciudad. Con ello se pretende que, además de

Tabla 1

Ciudades participantes en el proyecto $E M E C A M$, población y período a estudio

\begin{tabular}{|c|c|c|c|c|}
\hline \multirow{2}{*}{ Ciudad } & \multicolumn{3}{|c|}{ Población } & \multirow{2}{*}{ Periodo estudio } \\
\hline & Total & $\geq 70$ años $(\%)^{1}$ & Fuente & \\
\hline Barcelona & 1.643 .545 & $191.724(11,7 \%)$ & censo 1991 & $1991-1995$ \\
\hline Gran Bilbao $^{2}$ & 667.034 & $59.657(8,9 \%)$ & censo 1991 & $1 / 4 / 92-31 / 3 / 96$ \\
\hline Castellón & 134.213 & $11.229(8,4 \%)$ & censo 1991 & 1991-1995 \\
\hline Cartagena & 168.023 & $12.287(7,3 \%)$ & censo 1991 & $1990-1996$ \\
\hline Huelva & 142.547 & $9.281(6,5 \%)$ & censo 1996 & $26 / 10 / 93-26 / 10 / 96$ \\
\hline Sevilla & 683.028 & $52.637(7,7 \%)$ & censo 1996 & $31 / 8 / 92-10 / 6 / 96$ \\
\hline Madrid & 2.940 .896 & $321.297(10,9 \%)$ & censo 91 -padrón $96^{3}$ & 1992-1995 \\
\hline Pamplona $^{4}$ & 213.839 & $21.360(10,0 \%)$ & censo 91 -padrón $96^{3}$ & $1 / 4 / 91-31 / 3 / 95$ \\
\hline Valencia & 749.796 & $75.256(10,0 \%)$ & censo 91 -padrón $96^{3}$ & $1992-1996$ \\
\hline Vigo & 274.574 & $19.496(7,1 \%)$ & censo 1991 & 1991-1994 \\
\hline Vitoria & 214.148 & $16.483(7,7 \%)$ & censo 1991 & $1990-1994$ \\
\hline Gijón & 261.724 & $24.787(9,6 \%)$ & censo 91 -padrón $96^{3}$ & $1993-1996$ \\
\hline Oviedo & 198.050 & $19.267(9,8 \%)$ & censo 91 -padrón $96^{3}$ & $1993-1996$ \\
\hline Zaragoza & 572.212 & $53.304(9,3 \%)$ & censo 91 & $1991-1995$ \\
\hline TOTAL & 8.882 .834 & $888.065(10 \%)$ & & \\
\hline
\end{tabular}

\footnotetext{
' Porcentaje de la población $\geq 70$ años sobre el total.

" El Gran Bilbao incluye las poblaciones de Bilbao, Erandio, Leioa, Barakaido, Sestao, Portugalete y Saturtzi.

${ }^{3}$ Media aritmética del censo 1991 y el padrón 1996.

+ Pamplona más los municipios de Barañain y Burlada.
} 
la obtención de estimaciones válidas sobre el impacto de la contaminación atmosférica en la población urbana española, el proyecto sirva para el desarrollo de habilidades de investigación en todos los grupos y la adquisición de experiencia en la metodología de análisis empleada.

Así, los objetivos de cada subproyecto son:

1. Describir las variaciones temporales, tomando el día como unidad de análisis, de la mortalidad general y específica por causas respiratorias y del aparato circulatorio en la ciudad correspondiente, durante el período determinado.

2. Describir la situación actual y las variaciones temporales de los indicadores de contaminación atmosférica en la ciudad correspondiente durante dicho período.

3. Cuantificar la magnitud de la asociación entre la mortalidad general y específica y la variación de los niveles de contaminación atmosférica.

4. Además de lo anterior, los grupos de Valencia y Barcelona tienen establecidos unos objetivos específicos en relación con su papel de centro coordinador y de grupo que realizará el metaanálisis, respectivamente. Ambos grupos, además, realizan el asesoramiento en la metodología de análisis.

Para el conjunto del estudio multicéntrico los objetivos planteados son:

1. Elaborar y adaptar un protocolo estandarizado para el conjunto de grupos del proyecto.

2. Valorar el impacto agudo de la contaminación atmosférica en la mortalidad de la población urbana de las ciudades participantes.

3. Aplicar la metodología de análisis y el abordaje de meta-análisis para los estudios con datos de series temporales.

\section{MATERIAL Y MÉTODOS}

\section{Información}

En este proyecto se analizan los datos de las series temporales de mortalidad, contaminantes, temperatura y otros factores obtenidos a partir de registros de instituciones públicas. Los datos utilizados son:

- Mortalidad: Como variable respuesta se estudia el número de defunciones diarias entre los residentes de la ciudad correspondiente por las causas siguientes según la Clasificación Internacional de Enfermedades, 9. ${ }^{a}$ revisión $(\mathrm{CIE}-9)^{16}$ :

- Todas las causas excluyendo las externas (CIE-9, 001-799).

- Mortalidad por todas las causas excepto las externas en personas de 70 y más años

- Enfermedades del aparato circulatorio (CIE-9, 390-459).

- Enfermedades respiratorias (CIE-9, 460-519).

Para evitar sesgos de mala clasificación en cuanto a la exposición, se excluyeron las muertes de los residentes en el municipio si habían ocurrido fuera de la ciudad. En todas las ciudades se ba podido estudiar los grupos de causas contemplados en el protocolo. La tabla 2 muestra los descriptivos de la mortalidad diaria para cada ciudad.

- Contaminación atmosférica: los datos sobre contaminantes atmosféricos incluidos en el estudio proceden de las Redes de Vigilancia de cada una de las ciudades. Estas redes son gestionadas por los servicios de protección ambiental de las Comunidades Autónomas y/o los dependientes de los Ayuntamientos. Los contaminantes incluidos en nuestro estudio son los siguientes:

1. Valores medios de 24 horas: Partículas en suspensión (medidas como «humos negros»), partículas totales en suspensión 
Tabla 2

\begin{tabular}{|c|c|c|c|c|}
\hline \multicolumn{5}{|c|}{ Mortalidad diaria por ciudades y causa de defunción } \\
\hline \multirow{3}{*}{ Ciudad } & \multicolumn{4}{|c|}{ Grupos de causas } \\
\hline & $\begin{array}{c}\text { Todas menos las } \\
\text { externas } \\
(C I E-9<800)\end{array}$ & $\begin{array}{c}\text { Id en mayores de } \\
70 \text { años } \\
(C I E-9<800)\end{array}$ & $\begin{array}{l}\text { Respiratorias } \\
\text { (CIE-9 460-519) }\end{array}$ & $\begin{array}{l}\text { Aparato circulatorio } \\
\text { (CIE-9 390-459) }\end{array}$ \\
\hline & $\operatorname{Med}(D e s)^{*}$ & $\operatorname{Med}(D e s)^{*}$ & $\operatorname{Med}(D e s) *$ & $\operatorname{Med}(D e s) *$ \\
\hline Barcelona & $43,6(8,6)$ & $31,0(7,2)$ & $3,8(2,2)$ & $17,4(5,1)$ \\
\hline Gran Bilbao & $13,6(4,0)$ & $8,9(3,3)$ & $1,6(0,9)$ & $4,9(2,3)$ \\
\hline Castellón & $2,9(1,8)$ & $2,1(1,5)$ & $0,3(0,5)$ & $1,3(1,2)$ \\
\hline Cartagena & $3,5(2,0)$ & $2,5(1,6)$ & $0,4(0,6)$ & $1,5(1,3)$ \\
\hline Huelva & $2,5(1,6)$ & $1,7(1,3)$ & $0,3(0,5)$ & $1,0(1,0)$ \\
\hline Sevilla & $13,5(4,2)$ & $8,9(3,4)$ & $1,2(1,2)$ & $5,5(2,6)$ \\
\hline Madrid & $60,8(11,1)$ & $40,5(8,7)$ & $6,1(3,1)$ & $22,0(6,1)$ \\
\hline Pamplona & $4,9(3,7)$ & $3,6(3,3)$ & $0,5(0,8)$ & $1,8(2,1)$ \\
\hline Valencia & $16,4(4,8)$ & $11,3(3,9)$ & $1,5(1,3)$ & $6,6(2,9)$ \\
\hline Vigo & $5,3(2,4)$ & $3,5(2,0)$ & $0,5(0,7)$ & $2,0(1,5)$ \\
\hline Vitoria & $3,5(1,9)$ & $2,4(1,6)$ & $0,3(0,6)$ & $1,2(1,1)$ \\
\hline Gijón & $6,3(2,7)$ & $4,7(2,3)$ & $0,7(0,9)$ & $2,2(1,6)$ \\
\hline Oviedo & $4,5(2,2)$ & $3,3(1,9)$ & $0,5(0,7)$ & $1,5(1,3)$ \\
\hline Zaragoza & $12,5(3,9)$ & $9,0(3,2)$ & $1,3(1,2)$ & $4,6(2,3)$ \\
\hline
\end{tabular}

${ }^{*} \operatorname{Med}($ Des $)=$ media $\left(\mathrm{y}\right.$ desviación estándar) del n. ${ }^{\circ}$ de defunciones diarias; CIE-9: Clasificación Internacional de Enfermedades, 9.a revisión.

(PTS) o partículas con diámetro inferior a 10 micras $\left(\mathrm{PM}_{10}\right)$; dióxido de azufre $\left(\mathrm{SO}_{2}\right)$; dióxido de nitrógeno $\left(\mathrm{NO}_{2}\right)$ y monóxido de carbono (CO).

2. Valor máximo horario de $\mathrm{SO}_{2}, \mathrm{NO}_{2}$ partículas en suspensión

3. Valor máximo de 8 horas de ozono $\left(\mathrm{O}_{3}\right)$.

La mayoría de las ciudades (tabla 3) disponen de estaciones captadoras de la contaminación de tipo automático, las cuales permiten la medición de los niveles de contaminantes con la frecuencia que se requiera (habitualmente cada 15 minutos). En algunos casos, sin embargo, los datos de la red automática de vigilancia de la contaminación atmosférica se encuentran disponibles únicamente desde hace pocos años, como en el caso de Valencia, o no se ha podido disponer de una serie continuada de duración adecuada, como en Zaragoza. En otras ciudades, por lo general las de menos pobla- ción y sin concentración de industrias potencialmente contaminantes, únicamente se dispuso de los datos procedentes de la red manual de vigilancia de la contaminación atmosférica. En esta red únicamente se recogen de manera sistemática las partículas en suspensión y el $\mathrm{SO}_{2}$. En el caso de las partículas, el método de determinación utilizado es el análisis del humo normalizado (humos negros), que mide la concentración en la atmósfera de «humos» (partículas negras pequeñas que permanecen en suspensión en el aire y que proceden, en su mayor parte de las combustiones), y está basado en el ennegrecimiento producido en un papel de filtro, mediante reflectometría, cuando una muestra de aire pasa a través de él. Con este método se captan principalmente partículas con diámetro aerodinámico alrededor de 4-5 $\mu \mathrm{m}$, siendo mucho menor la proporción de partículas con diámetro superior a 7-9 $\mu \mathrm{m}$. En cuanto a la determinación del $\mathrm{SO}_{2}$ presente en la atmósfera se realiza mediante espectrofotometría, con el método de la thorina ${ }^{17}$. Los métodos de determinación 
Tabla 3

Contaminantes estudiados en cada una de las ciudades

\begin{tabular}{|c|c|c|c|}
\hline \multirow{2}{*}{ Ciudad } & \multicolumn{3}{|c|}{ Contaminantes } \\
\hline & Valores medios de 24 horas & Valor máximo horario & Valor máximo de 8 horas \\
\hline Barcelona & $\mathrm{HN}, \mathrm{PTS}, \mathrm{SO}_{2}, \mathrm{NO}_{2}, \mathrm{CO}$ & $\mathrm{SO}_{2}, \mathrm{NO}_{2}$ & $\mathrm{O}_{3}$ \\
\hline Gran Bilbao & $\mathrm{HN}, \mathrm{PTS}, \mathrm{SO}_{2}, \mathrm{NO}_{2}$ & PTS, $\mathrm{SO}_{2}, \mathrm{NO}_{2}$ & \\
\hline Castellón & $\mathrm{HN}, \mathrm{SO}_{2}$ & & \\
\hline Cartagena & PTS, $\mathrm{SO}_{2}$ & PTS, $\mathrm{SO}_{2}$ & \\
\hline Huelva & $\mathrm{PM}_{10}, \mathrm{SO}_{2}, \mathrm{NO}_{2}, \mathrm{CO}$ & & \\
\hline Sevilla & $\mathrm{PM}_{10, \mathrm{so} 2}, \mathrm{NO}_{2}$ & & \\
\hline Madrid & $\mathrm{PM}_{10}, \mathrm{SO}_{2}, \mathrm{NO}_{2}, \mathrm{CO}$ & $\mathrm{SO}_{2}, \mathrm{NO}_{2}$ & $\mathrm{O}_{3}$ \\
\hline Pamplona & $\mathrm{HN}, \mathrm{SO}_{2}$ & & \\
\hline Valencia & $\mathrm{HN}, \mathrm{SO}_{2}, \mathrm{NO}_{2}, \mathrm{CO}$ & $\mathrm{SO}_{2}, \mathrm{NO}_{2}$ & $\mathrm{O}_{3}$ \\
\hline Vigo & $\mathrm{HN}, \mathrm{SO}_{2}$ & & \\
\hline Vitoria & $\mathrm{HN}, \mathrm{SO}_{2}$ & & \\
\hline Gijón & $\mathrm{HN}, \mathrm{PTS}, \mathrm{SO}_{2}, \mathrm{NO}_{2}, \mathrm{CO}$ & $\mathrm{SO}_{2}, \mathrm{NO}_{2}$ & \\
\hline Oviedo & $\mathrm{HN}, \mathrm{PTS}, \mathrm{SO}_{2}, \mathrm{NO}_{2}, \mathrm{CO}$ & $\mathrm{SO}_{2}, \mathrm{NO}_{2}$ & \\
\hline Zaragoza & $\mathrm{HN}, \mathrm{SO}_{2}$ & & \\
\hline
\end{tabular}

HN: humos negros.

PTS: partículas totales en suspensión.

de los contaminantes medidos en la red automática son: la radiación beta para las partículas totales, la quimiluminiscencia para el $\mathrm{NO}_{2}$, la fluorescencia ultravioleta en el caso del $\mathrm{SO}_{2}$, la absorción ultravioleta en la determinación de $\mathrm{O}_{3} \mathrm{y}$, por último, la absorción infrarrojo para el $\mathrm{CO}^{18}$. Las características de los equipos captadores están determinadas por la normativa vigente, debiendo cumplir una serie de condiciones ${ }^{17}$. Además, las distintas redes urbanas de vigilancia de la contaminación atmosférica están coordinadas en un único sistema nacional. Esta circunstancia aporta confianza de cara a la comparabilidad de las mediciones de los contaminantes.

Por otro lado, el protocolo de nuestro estudio incluye una serie de guías, siguiendo lo establecido en el proyecto $A P H E A^{9}$, para la obtención de las variables de contaminación atmosférica. En este sentido, la exposición diaria de la población se ha estimado usando, en cada caso, el promedio de los datos de las estaciones captadoras según los siguientes criterios:
- Admisibilidad de las estaciones captadoras: Se estudian únicamente las estaciones urbanas, no incluyéndose las situadas fuera de las ciudades ni las que miden la contaminación «de fondo». En cierto sentido, el Gran Bilbao y Pamplona constituyen una excepción, al tratarse de grupos de municipios; sin embargo, los captadores incluidos se hallan situados en los núcleos urbanos que, además, están muy próximos entre sí. Se excluyeron del análisis las mediciones procedentes de estaciones captadoras situadas en autopistas de acceso limitado.

- Porcentaje de datos válidos: En los cálculos del promedio de cada contaminante se incluyeron sólo los valores de las estaciones captadoras que presentaron un porcentaje de datos válidos mayor al $75 \%$ de días para todo el período a estudio. En los cálculos de la media de $1 \mathrm{~h}$ y $24 \mathrm{~h}$ (cuando los datos provienen de estaciones automáticas) se requiere un mínimo del $75 \%$ de datos válidos. Se desecharon los datos procedentes de captadores en los que, a pesar de cumplir con el criterio de porcentaje de datos váli- 
dos, los valores perdidos se presentaban en unos determinados períodos de tiempo (por ejemplo fines de semana, vacaciones o durante el verano).

- Número de estaciones captadoras: Se estimó conveniente un mínimo de tres estaciones válidas por contaminante. En algunos casos límite se estudió la posibilidad de la inclusión de indicadores con datos de dos estaciones. En ningún caso se han incluido en el estudio valores de un contaminante a partir de los datos de una sola estación.

- Imputación de datos perdidos: Los valores perdidos de las estaciones incluidas se corrigieron para el cálculo del promedio diario con los valores obtenidos en la regresión de cada una de ellas utilizando los datos de las demás estaciones captadoras como variables explicativas.

En los trabajos correspondientes de los grupos participantes en el proyecto $E M E$ $C A M$ se detallan el número de captadores seleccionado por contaminante, la correlación entre los mismos y los valores obtenidos en cada una de las ciudades.

\section{Variables de control}

- Estacionalidad y otras variables cronológicas: La estacionalidad (anual, semestral, trimestral, mensual o semanal) y la tendencia son variables por las que es preciso controlar en los estudios de series temporales (ver apartado de análisis) ${ }^{11,19}$. También se tuvieron en cuenta otros sucesos relacionados con el calendario, como los días festivos (sin contar los domingos, es decir fiestas nacionales, autonómicas y locales) y los días inusuales o extraordinarios (huelga médica o grandes acontecimientos, como la Expo-92 en Sevilla o los Juegos Olímpicos en Barcelona).

- Variables meteorológicas: Después de la estacionalidad éstas son las variables más importantes de modelizar, cuando se analiza la asociación de la mortalidad con los contaminantes atmosféricos por su posible efecto confusor $^{11,19}$. Los valores medios diarios de la temperatura media (calculada como el promedio de las temperaturas máxima y mínima) y la humedad relativa (calculada como el promedio de los valores de humedad relativa en diferentes momentos del día: $0,7,13$ y 18 horas) fueron las variables incluidas en el estudio (tabla 4).

- Incidencia de gripe: obtenida a partir del número de casos semanales de gripe notificados al sistema de Enfermedades de Declaración Obligatoria. Dicho número se dividió por 7 para tener una aproximación del número de casos diarios.

\section{ANÁLISIS DE LA INFORMACIÓN}

El análisis epidemiológico de los datos se ha desarrollado siguiendo un proceso paulatino que complementa el análisis simple con el uso de modelos multivariantes. Se consideró necesario, en una primera etapa, la realización de un estudio descriptivo. Esta etapa incluyó el estudio bivariado entre las variables por medio de las matrices de correlaciones y gráficos diagnósticos. Especialmente útiles fueron las representaciones gráficas, como las secuencias de las series, los diagramas de dispersión, las funciones de autocorrelación y las de correlaciones cruzadas. En los diagramas de dispersión, la forma de la relación entre la mortalidad y cada una de las variables a estudio se valoró mediante un método no paramétrico de regresión ponderado, lowess (por las siglas en inglés) ${ }^{20}$. La filosofia subyacente a esta aproximación es la de dejar que sean los propios datos los que muestren la forma funcional adecuada, sin proponer ninguna forma previa de dependencia entre la variable resultado y las variables predictoras ${ }^{21}$. Por último, esta primera etapa se complementó con el análisis simple de Poisson de la relación entre las series de mortalidad y las de los distintos contaminantes a estudio en cada una de las ciudades. 
Tabla 4

Datos meteorológicos: promedios diarios de temperatura y humedad relativa

\begin{tabular}{|c|c|c|c|c|c|c|c|c|}
\hline \multirow{3}{*}{ Ciudad } & \multicolumn{6}{|c|}{ Período } & \multirow{2}{*}{\multicolumn{2}{|c|}{$\begin{array}{c}\text { Indicador } \\
\text { de estacionalidad }\end{array}$}} \\
\hline & \multicolumn{2}{|c|}{ Todo el periodo } & \multicolumn{2}{|c|}{ Semestre cálidol } & \multicolumn{2}{|c|}{ Semestre frio ${ }^{2}$} & & \\
\hline & Temp $p^{3}$ & $\mathrm{Hum}^{4}$ & Temp $p^{3}$ & $\mathrm{Hum}^{4}$ & Temp $p^{3}$ & $\mathrm{Hum}^{4}$ & Temp 5 & Hum $^{6}$ \\
\hline Barcelona & $16,5(5,8)$ & $71,5(5,3)$ & $21,0(4,2)$ & $71,3(5,7)$ & $11,7(2,7)$ & $71,8(4,9)$ & 9,3 & 1,01 \\
\hline Gran Bilbao & $15,2(4,7)$ & $80,0(10,3)$ & $18,6(3,4)$ & $81,3(9,8)$ & $11,8(3,2)$ & $78,8(10,7)$ & 6,8 & 0,96 \\
\hline Castellón & $17,2(5,8)$ & $70,7(12,0)$ & $21,9(3,7)$ & $70,0(10,5)$ & $12,5(3,0)$ & $71,3(13,3)$ & 9,4 & 1,01 \\
\hline Cartagena & $19,0(5,3)$ & $75,3(12,1)$ & $23,2(3,3)$ & $75,5(11,1)$ & $14,9(2,8)$ & $75,0(13,8)$ & 8,3 & 0,99 \\
\hline Huelva & $18,3(5,5)$ & $64,5(20,6)$ & $22,7(3,5)$ & $54,0(22,0)$ & $13,9(3,2)$ & $75,2(12,0)$ & 8,8 & 1,40 \\
\hline Sevilla & $18,4(6,1)$ & $64,4(14,5)$ & $23,4(4,3)$ & $57,9(12,8)$ & $14,1(3,4)$ & $70,3(13,3)$ & 9,3 & 1,21 \\
\hline Madrid & $14,4(7,7)$ & $61,8(16,7)$ & $20,3(5,4)$ & $53,8(14,2)$ & $8,4(4,1)$ & $69,9(15,0)$ & 11,9 & 1,30 \\
\hline Pamplona & $12,9(6,5)$ & $68,7(12,6)$ & $17,5(5,0)$ & $65,9(10,8)$ & $7,5(3,5)$ & $73,9(12,0)$ & 9,9 & 1,12 \\
\hline Valencia & $18,2(5,5)$ & $64,8(13,6)$ & $22,9(3,4)$ & $65,3(12,8)$ & $14,3(3,0)$ & $61,0(14,4)$ & 8,6 & 0,93 \\
\hline Vigo & $13,5(4,7)$ & $77,3(12,2)$ & $16,9(3, T)$ & $75,1(12,3)$ & $10,1(2,8)$ & $79,5(11,7)$ & 6,8 & 1,06 \\
\hline Vitoria & $11,6(6,2)$ & $76,5(11,1)$ & $16,1(4,5)$ & $72,8(10,8)$ & $6,9(3,6)$ & $80,2(10,2)$ & 9,2 & 1,10 \\
\hline Gijón & $13,8(4,2)$ & $79,4(9,3)$ & $17,4(2,7)$ & $80,3(8,1)$ & $10,6(2,6)$ & $78,6(10,3)$ & 6,8 & 0,97 \\
\hline Oviedo & $13,2(4,5)$ & $78,0(11,2)$ & $16,4(3,1)$ & $79,6(9,7)$ & $10,0(3,2)$ & $76,3(12,4)$ & 6,4 & 0,95 \\
\hline Zaragoza & $15,5(7,3)$ & $64,5(14,9)$ & $21,1(5,1)$ & $57,7(12,4)$ & $9,7(4,0)$ & $71,3(14,2)$ & 11,4 & 1,23 \\
\hline
\end{tabular}

${ }^{1}$ Semestre cálido: meses de mayo a octubre.

${ }^{2}$ Semestre friv: meses de noviembre a abril.

${ }^{3}$ Temp: temperatura media diaria $\left(e{ }^{\circ} \mathrm{C}\right.$ ), y su desviación estándar.

${ }^{4}$ Hum: humedad relativa diaria (en \%) (media de los valores a las $0,7,13$ y 18 horas, excepto Valencia que es la media de los valores a las $7,13,18$ horas), y su desviación estándar.

${ }^{5}$ Diferencia: Temperatura media meses cálidos - temperatura media meses fríos.

${ }^{6}$ Razón: Humedad relativa meses fríos / Humedad relativa meses cálidos.

\section{Análisis multivariante}

Desde el punto de vista de la distribución se asume que la mortalidad diaria se distribuye según un proceso de Poisson. Este proceso no es estacionario, esto es, el riesgo de muerte varía con algunas variables predictoras. En este estudio la unidad de observación es el día. Una serie temporal es una sucesión ordenada en el tiempo de valores de una variable $^{22}$. En series temporales con datos día a día los factores de confusión son variables que cambian a lo largo del tiempo. Tales factores pueden ser, además de las variables meteorológicas a estudio, las epidemias de ciertas enfermedades (gripe) o la diferente ocurrencia de las variables según días de la semana y festivos. Además, se han de tener en cuenta los componentes de las series temporales: estacionalidad, tendencia y cambios anuales. Otras variables como edad, hábito tabáquico o profesión no influirán en las variaciones diarias de la mortalidad dado que la exposición a dichos factores no cambia en la población día a día. Por lo demás, el efecto del tabaco y de otros muchos tóxicos propios del ambiente de trabajo se evidencian a largo plazo. Esta es la gran ventaja de los estudios ecológicos temporales sobre los geográficos. En un artículo de este mismo número se exponen más detalladamente los fundamentos metodológicos del análisis de series temporales en epidemiología ambiental ${ }^{19}$.

El procedimiento de análisis seguido para valorar la relación entre mortalidad y las variables explicativas se basó principalmente en la metodología desarrollada en el proyecto $A P H E A^{10}$. Durante la primera etapa de nuestro proyecto se siguió una estrategia idéntica a la del proyecto europeo. Dicho análisis consta básicamente de dos fases: la primera, de identificación de un modelo basal para cada una de las causas específicas de muerte y el grupo de edad estudiado, ajustando por mínimos cuadrados un mode- 
lo de regresión gaussiano para, posteriormente, realizar una estimación cuantitativa de la asociación de los contaminantes y la mortalidad por medio de la regresión de Poisson. Para la construcción del modelo mediante mínimos cuadrados la variable mortalidad se aproximaba a una normal, usando transformación logarítmica.

En una segunda etapa del desarrollo del proyecto se consideró conveniente introducir algunas modificaciones a dicho procedimiento, ya que nuestro estudio presenta algunas diferencias destacables con respecto al proyecto $A P H E A$. Por un lado, gracias a la experiencia derivada de dicho proyecto, se contaba con un protocolo muy bien definido que permitía establecer la secuencia de los procedimientos siguiendo un criterio estadístico. Por otro lado, el tamaño de la población de las ciudades participantes en el proyecto $E M E C A M$ es, en promedio, bastante menor que el de las ciudades participantes en el proyecto APHEA. Por esta razón el número de defunciones diarias, especialmente en las series de mortalidad por causas, era pequeño y en bastantes días no se observó ninguna defunción. Por tanto, su adaptación a una distribución normal es cuestionable y el ajuste de una regresión gaussiana no es del todo adecuado. Por todo ello, se decidió realizar tanto la identificación del modelo basal como la estimación de la asociación con los contaminantes utilizando regresión de Poisson. Con esta adaptación del protocolo consideramos que, en nuestro caso, el análisis se adapta mejor a la distribución de la serie, se simplifica su realización y se evita una fuente de errores, al omitir un paso (regresión lineal múltiple), que podía dejar fuera del modelo a variables que podrían ser significativas en el modelo Poisson.

En otro trabajo ${ }^{23}$ se detallan los pasos establecidos en el protocolo del proyecto. Asimismo, se ilustra su aplicación mediante la realización de un ejemplo. Brevemente, consiste en los dos pasos siguientes utilizando en ambos regresión de Poisson:
- Construcción de un modelo basal para cada una de las causas de muerte estudiadas. Los factores que se tienen en cuenta para controlar la confusión son las variables temporales (estacionalidad, tendencia, cambios anuales) y de calendario (día de la semana, festivos), los fenómenos meteorológicos (temperatura media y humedad relativa diarias), la incidencia de gripe y otros eventos (huelgas, acontecimientos especiales). Los efectos de las variables meteorológicas y de la gripe pueden ser inmediatos u ocurrir con algún retraso. Por ejemplo, las bajas temperaturas pueden tener un efecto sobre la mortalidad el mismo día o al día siguiente o unos días después. Por ello, se examina el efecto retardado de las variables meteorológicas hasta una semana antes de la fecha de defunción y el de la gripe hasta quince días antes.

- Estimación de la asociación entre cada uno de los contaminantes y la mortalidad. A los modelos construidos en la etapa anterior se les añaden las variables de contaminación atmosférica. Se introducen los contaminantes de uno en uno, con el fin de evitar problemas de colinealidad. Al igual que en el caso de las variables meteorológicas y la gripe, los efectos retardados deben ser investigados también para el caso de los contaminantes atmosfćricos. Una cuestión que se plantea es hasta dónde llegar en la comprobación de los retardos. Siguiendo a Schwartz et al ${ }^{11}$, se ha considerado que explorar demasiados términos de retardos puede llevar a encontrar una asociación espúrea. En ese sentido, nos parece sensato no explorar los retardos más allá del quinto día previo a la defunción. Entre los retardos probados se selecciona el de mejor ajuste para cada contaminante y su asociación se expresa como el riesgo relativo de morir por incremento de $10 \mu \mathrm{g} / \mathrm{m}^{3}$ (excepto en el caso del $\mathrm{CO}$ que se expresa como incremento de $1 \mathrm{mg} / \mathrm{m}^{3}$ ). Con el fin de comparar el efecto de los distintos contaminantes en cada ciudad, también se calcula el efecto relativo a un incremento igual a la diferencia del percentil 90 al percentil 10 . 
En todos los modelos construidos se diagnostica la posible autocorrelación de los residuos. El que alguno de ellos presente este fenómeno indica una incompleta o inadecuada especificación de la asociación, motivo por el que se introducen los términos autorregresivos, es decir los retardos de mortalidad correspondientes en el modelo.

Por último, se realizan una serie de análisis complementarios que incluyen el estratificado según período (meses cálidos o fríos) y la investigación de posibles interacciones entre los posibles contaminantes y entre éstos y la temperatura. También se llevan a cabo análisis de sensibilidad para valorar la estabilidad de las posibles asociaciones encontradas.

\section{Meta-análisis}

Con los riesgos relativos obtenidos en cada ciudad y para cada contaminante se llevará a cabo un meta-análisis. En concreto, se realizará una media ponderada de los coeficientes de regresión «locales» (esto es, proporcionados por cada uno de los centros). Asimismo, se contrastará la homogeneidad de los coeficientes utilizando el contraste ji cuadrado $\left(\chi^{2}\right)$. Si la hipótesis de homogeneidad se rechazase se utilizaría un modclo de cfectos aleatorios. En caso de existir, la heterogeneidad será explicada utilizando una regresión ponderada de los coeficientes de regresión locales sobre variables explicativas fijas en el tiempo. Se considerarán, entre otras, las siguientes variables explicativas: indicadores de la salud general de la población (tasa estandarizada de la mortalidad, esperanza de vida, proporción de ancianos, prevalencias de enfermedades respiratorias y tabaquismo, etcétera); variables meteorológicas por estación; indicadores de la bondad de los datos (correlación entre estaciones captadoras, número de ha- bitantes en torno a las mismas, origen de los datos de mortalidad, etcétera).

El meta-análisis, en este contexto, difiere en aspectos esenciales de los meta-análisis habituales que se realizan a partir de resultados publicados y que se realizan únicamente cuando existe un número importante de artículos con relación a una hipótesis. Como consecuencia, los problemas habituales de los meta-análisis no deben ocurrir en este estudio. Como en el caso de los meta-análisis llevados a cabo en el proyecto APHEA ${ }^{24,25}$ : no habrá sesgo de publicación (se incluirán todos los resultados en el estudio), no habrá sesgo de selección (todas las ciudades que pueden aportar la información necesaria y desean entrar en el proyecto se incluyen en él mucho antes de conocer los resultados); una vez finalizado el estudio, toda la información se encontrará disponible para posibles reanálisis.

El grupo EMECAM lo forman: F Ballester, S Pérez-Hoyos, JM Tenías, R Molina, J González-Aracil (Valencia, Centro coordinador); M Saez, MA Barceló, C Saurina, A 'Tobias (Barcelona); E. Alonso, K. Cambra (Bilbao); M Taracido, JM Barros, I Castro, A Figueiras, A Montes, E Smyth (Vigo); JM Ordóñez, E Aranguez, I Galán, AM Gandarillas (Madrid); I Aguinaga, MY Floristan, F Guillén, MS Laborda, MA Martínez, MT Martínez, PJ Oviedo (Pamplona); A Daponte, R Garrido de la Sierra, JL Gurucelain, P. Gutiérrez, JA Maldonado, JL Martín, JM Mayoral, R Ocaña, J Serrano (Granada); JB Bellido, A Arnedo, F González (Castellón); JJ Guillén, Ll Cirera, L García, E Jiménez, MJ Martínez, S Moreno, C Navarro (Cartagena); MJ Pérez, A Alonso, JJ Estíbalez, MA García-Calabuig, (Vitoria); A Cañada, C Fernández, F Fernández, V García, I Huerta, V Rodríguez (Asturias); F Arribas, M Navarro, C Martos, MJ Rabanaque, E Muniesa, JM Abad, JI Urraca (Zaragoza); y J Sunyer como asesor del proyecto. 


\section{BIBLIOGRAFÍA}

1. Holland WW, Bennett AE, Cameron IR, Florey $\mathrm{CV}$, Leeder SR, Schilling RSF et al. Health effects of particulate pollution: reappraising the evidence. Am J Epidemiol 1979; 110: 525-659.

2. Air Quality Guidelines for Europe. World Health Organization. Regional Office for Europe. WHO Regional Publications, European Series No. 23. Copenhagen, 1987.

3. Momas I, Pirard P, Quenel P, Medina S, Le Moullec Y, Ferry R et al. Pollution atmosphérique urbaine et mortalité: une synthèse des études épidemiologiques publiées entre 1980 et 1991. Rev Epidemiol Santć Publique.

4. Schwartz J, Marcus A. Mortality and air pollution in London: a time series analysis. Am J Epidemiol 1990; 131: 185-94.

5. Schwartz J. Air Pollution and Daily mortality in Birminghan, Alabama. Am J Epidemiol 1993; 137: 1136-47.

6. Touloumi G. Pocock SJ, Katsouyanni K, Trichopoulos D. Short-Term Effects of Air Pollution on Daily Mortality in Athenas: A Time Series Analysis. Int J Epidemiol 1994; 23:957-67.

7. Sunyer J, Antó JM, Murillo C, Sáez M. Effects of urban air pollution on emergency room admissions for chronic obstructive pulmonary disease. Am J Epidemiol 1991; 134: 277-86.

8. Brunekeef B, Dockery DW, Krzyzanowsky M. Epidemiologic Studies on Short-Term Effects of Low Levels of Major Ambient Air Pollution Components. Environ Health Perspect 103 1995;Suppl 2:2-13.

9. Katsouyanni K, Zmirou D, Spix C, Sunyer J, Schoten JP, Pönka A et al. Short-term effects of air pollution on health: A european approach using epidemiologic time series data. The APHEA project: background, objectives, design. Eur Respir J 1995;8:1030-8.

10. Katsouyanni K, Schwartz J, Spix C, Touloumi G, Zmirou D, Zanobetti A et al. Short-term effects of air pollution on health: a European approach using epidemiological time-series data. The APHEA protocol. J Epidemiol Community Health 1996; 50 (Sup I):S12-S18.

11. Schwartz J, Spix C, Touloumi (i, Racharova I, Barumamdzdeh T, Le Tertre A et al. Methodological issues in studies of air pollution and daily counts of deaths and hospital admissions. J Epidemiol Community Health 1996; 50 (Sup I): S3-S11.

12. Sunyer J, Castellsagué J, Sáez M, Tobias A, Antó JM. Air pollution and mortality in Barcelona. J Epidemiol Community Health 1996; 50 (Sup I): S76-S80.
13. Ballester F, Corella D, Pérez-Hoyos S, Hervás A. Air pollution and mortality in Valencia (Spain): a study using the APHEA methodology. J Epidemiol Community Health 1996; 50: 527-33.

14. Alonso E, Cambra K, Urtiaga M. Análisis del episodio de disnea súbita ocurrido por emisión masiva de $\mathrm{SO}_{2}$ en Barakaldo (Bizkaia). Gac Sanit 1995, 9 (Sup I); 54.

15. Guillén JJ, Guillén F, Medrano J, García-Marcos L, Aguinaga I, Ñíguez JC. Afluencia inusual por asma y enfermedad pulmonar crónica en urgencias hospitalarias y contaminación por $\mathrm{SO}_{2}$ en Cartagena. Rev Esp Salud Pública 1995; 69: 305-14.

16. Organización Mundial de la Salud. Manual de la Clasificación Estadística Internacional de Enfermedades, Traumatismos y Causas de Defunción. 9. ${ }^{a}$ revisión. Publicación n. ${ }^{\circ} 353$. Washington: Organización Panamericana de Salud; 1978.

17. Ministerio de Sanidad y Consumo. Red Nacional de Vigilancia y Prevención de la Contaminación Atmosférica. Colección Sanidad Ambiental. Madrid: Ministerio de Sanidad y Consumo;1988.

18. Ministerio de Obras Públicas, Transportes y Medio Ambiente (MOPTMA). Estaciones y redes de vigilancia de la contaminación atmosférica en España. Descripción técnica y gráfica. Serie Monografias. Madrid: MOPTMA; 1993.

19. Sáez M, Pérez-Hoyos $S$, Tobías $A$, Saurina $C$, Barceló MA, Ballester F. Métodos de series temporales en los estudios epidemiológicos sobre contaminación atmosférica. Rev Esp Salud Pública $1999 ; 73: 133-143$.

20. Sánchez-Cantalejo E, Ocaña-Riol R. Actualizaciones en regresión: suavizando las relaciones. Gac Sanit 1997; 11:24-32.

21. Hastie TJ, Tibshirani RJ. Generalized Additive Models. Londres: Chapman and Hall; 1990: 9.

22. Murillo C. Métodos estadísticos de scries temporales. Aplicaciones sanitarias. Barcelona: SG;1994.p.17.

23. Pérez-Hoyos S, Sáez M, Barceló MA, Cambra K, Figueiras A, Aránguez E, et al. Protocolo EMECAM: Análisis del efecto a corto plazo de la contaminación atmosférica sobre la mortalidad. Rev Esp Salud Pública 1999; 73:177-185.

24. Katsouyanni K, Toulomi G, Spix C, Schwartz J, Balducci F, Medina S, et al. Short-term effects of ambient sulphur dioxide and particulate matter on mortality in 12 European cities: results from time series data from the APHEA project. BMJ 1997;314:1658-63.

25. Toulomi G, Katsouyanni K, Zmirou D, Schwartz J, Spix C, Ponce de Leon A, et al. Short-term effects of ambient oxidant exposure on mortality: a combined analysis within the APHEA project. Am J Epidemiol 1997;146:177-85. 